\title{
Atomic Force Microscopy Combined with Infrared Spectroscopy as a Tool to Probe Single Bacterium Chemistry
}

\author{
Kamila Kochan ${ }^{1}$, Anton Y. Peleg ${ }^{2,3}$, Philip Heraud ${ }^{1,2}$, Bayden R. Wood ${ }^{1}$ \\ ${ }^{1}$ Centre for Biospectroscopy and School of Chemistry, Monash University ${ }^{2}$ Infection and Immunity Program, Monash Biomedicine Discovery Institute and \\ Department of Microbiology, Monash University ${ }^{3}$ Department of Infectious Diseases, The Alfred Hospital and Central Clinical School, Monash University
}

\section{Corresponding Author}

Kamila Kochan

kamila.kochan@monash.edu

\section{Citation}

Kochan, K., Peleg, A.Y., Heraud, P., Wood, B.R. Atomic Force Microscopy Combined with Infrared Spectroscopy as a Tool to Probe Single Bacterium Chemistry. J. Vis. Exp. (163), e61728, doi:10.3791/61728 (2020).

\section{Date Published}

September 15, 2020

DOI

$10.3791 / 61728$

URL

jove.com/video/61728

\section{Abstract}

Atomic Force Microscopy-Infrared Spectroscopy (AFM-IR) is a novel combinatory technique, enabling simultaneous characterization of physical properties and chemical composition of sample with nanoscale resolution. By combining AFM with IR, the spatial resolution limitation of conventional IR is overcome, enabling a resolution of $20-100 \mathrm{~nm}$ to be achieved. This opens the door for a broad array of new applications of IR toward probing samples smaller than several micrometers, previously unachievable by means of conventional IR microscopy. AFM-IR is eminently suited for bacterial research, providing both spectral and spatial information at the single cell and intracellular level. The increasing global health concerns and unfavorable future prediction regarding bacterial infections, and especially, rapid development of antimicrobial resistance, has created an urgent need for a research tool capable of phenotypic probing at the single cell and subcellular level. AFM-IR offers the potential to address this need, by enabling detail characterization of chemical composition of a single bacterium. Here, we provide a complete protocol for sample preparation and data acquisition of single spectra and mapping modality, for the application of AFMIR toward bacterial studies.

\section{Introduction}

Bacteria are single cell prokaryotic organisms, occurring in various shapes and sizes, typically in the range of several hundred nanometers to micrometers. They exist in a variety of habitats and are essential to the existence of life. Within the human body, the majority of bacteria present in the gut are harmless and many are in fact beneficial ${ }^{1}$.
However, several bacterial species are pathogenic and cause a range of infectious diseases. Bacterial infections can lead to the development of sepsis and septic shock: a lifethreatening condition, resulting from the body's response to an infection ${ }^{2}$. Sepsis is a global major health threat, with high prevalence worldwide and severe mortality rates. In 
2017 alone, an estimated 50 million cases of sepsis were recorded worldwide, with 11 million of those resulting in death (approximately 20\%) $)^{2}$. Furthermore, a decrease in patient's survival chances, due to delayed therapy, was shown to occur in an hourly manner ${ }^{3,4}$.

Bacterial infections are treated with antibiotics. The severity of potential consequences of bacterial bloodstream infections (BSIs), together with a clear significance of quick initiation of antimicrobial therapy, prompt the need for immediate antibiotics administration. However, as the current diagnostic approaches used in clinical practice (e.g., blood culturing) require a relatively long time, antibiotics administration often occurs prior to positive BSI diagnosis ${ }^{5}$. This factor leads to extensive overuse of antibiotics, which-together with excessive antibiotic use in other sectors such as agriculture-creates a severe evolutionary pressure towards the development of antimicrobial resistance $(\mathrm{AMR})^{6,7}$. AMR is currently one of the most pressing global health issues $^{7,8}$ and, by 2050 , is predicted to become the leading cause of death ${ }^{9}$. The development of resistance, together with the spread of AMR strains is occurring at an alarming pace ${ }^{7,8,9}$ and exceeds, by far, the rate of discovery of new antibiotics ${ }^{10}$. New resistant phenotypes are continuously emerging worldwide, while

research dedicated towards understanding the AMRrelated changes is often slow and limited by available approaches ${ }^{11}$. In addition, the commonly used methods, such as polymerase chain reaction (PCR) and whole gene sequencing (WGS), focus only on genotypic changes. These are not sufficient to reveal the mechanisms of resistance ${ }^{11}$, prompting an urgent need for a research tool enabling to understand the chemical composition of bacteria.
Infrared spectroscopy (IR) provides a molecular characterization of the sample and thus is a promising candidate for phenotypic bacterial probing. Since its early applications $^{12}$, a great magnitude of examples of its use was demonstrated in the literature ${ }^{13,14}$. These include phenotypic-based identification of bacteria on genus ${ }^{15}$, species $^{16}$, and $\operatorname{strain}^{17,18}$ level. However, the spatial resolution of conventional IR is restricted to several microns due to the wavelength diffraction spatial resolution limit ${ }^{19}$. Since the size of majority of bacteria lies below that limit (e.g., Staphylococcus aureus $\approx 400 \mathrm{~nm}$ in diameter), conventional IR is not applicable for probing at the single-cell or intracellular level.

The spatial resolution limitation was recently overcome by combining IR spectroscopy with Atomic Force Microscopy (AFM-IR). In this instance, the IR absorption is detected indirectly, through thermal expansion of the material $^{19,20,21,22}$. In brief, the absorption of IR radiation results in a local temperature increase. This can be measured either directly ${ }^{23}$ or through the measurement of oscillation of the AFM cantilever probe, resulting from force impulse created by IR absorption 20,21 . The combinatory AFM-IR technique enables to achieve spatial resolution approaching $20 \mathrm{~nm}$, providing simultaneous information about local physical properties of a sample (AFM) and its chemical composition (AFM-IR). Collection of both, single spectra from selected spots and mapping of the intensity of selected wavenumber values within a chosen area are possible.

Considering the achievable spatial resolution of AFM-IR, it is evident that the technique opens the possibility of chemical/phenotypic probing of single bacterium cell and their intracellular composition ${ }^{24}$. Hitherto, several examples of the application of AFM-IR for single bacteria were 
demonstrated in the literature $19,20,21,22,25,26,27,28$. These involve single spectral analysis ${ }^{19,21,22}$ and mapping at the subcellular level $19,22,25,26,27,28$. For example, the ability to detect intracellular lipid vesicles ${ }^{27}$ and viruses ${ }^{28}$ within single bacterium has been described. These results demonstrate the usefulness of AFM-IR for nanoscale studies of single bacteria and clinically relevant pathogens ${ }^{19}$.

Hence, we present a sample preparation and collection method for AFM-IR data of multilayer, monolayer and single cell bacterial samples. The protocol described herein was applied to study different species of bacteria ${ }^{22}$ and the changes in their chemical composition. In particular, the in vivo development of vancomycin resistance and daptomycin non-susceptibility was investigated in clinical pairs of $S$. aureus $^{19}$. Both, vancomycin intermittent resistance and daptomycin non-susceptibility in S. aureus (VISA and DpR) emerged relatively recently, following the increased use and introduction of these antibiotics to clinics, constituting a significant medical problem. Furthermore, in particular, the mechanism of daptomycin non-susceptibility still remains elusive, impeding alternative drug development ${ }^{19,29}$. The presented protocol focuses on provision of reliable AFM-IR spectra of single bacteria, which can further be analyzed using a variety of chemometric approaches, according to the experimental aims. It additionally includes the mapping approach, which is applicable for intra-cellular studies.

\section{Protocol}

All work conducted with pathogenic bacteria should be undertaken with appropriate safety measures in place. These include working in a laboratory with adequate biosafety level and in a biosafety cabin (PC2) as well as careful decontamination of work area with an appropriate disinfectant, e.g., 80\% ethanol solution. Appropriate PPE must be worn all the time.

\section{Preparation of solvents and materials}

1. Solvents: Use ultrapure water as a solvent. Use purified water, autoclaved prior to the experiment to avoid any potential cross-contamination.

2. Substrate: Use any of these substrates for AFM-IR, e.g., $\mathrm{ZnSe}, \mathrm{CaF}_{2}, \mathrm{BaF}_{2}$, etc. Since AFM-IR is, in principle, a non-destructive technique, one can apply a variety of other research tools to the same sample post AFMIR analysis. For instance, correlation of the results with Raman spectroscopy can be performed if Raman grade $\mathrm{CaF}_{2}$ or $\mathrm{BaF}_{2}$ slides are used.

3. Use glass vials instead of plastic tubes as plastic can contaminate the sample.

\section{Sample preparation for AFM-IR}

1. Growth/incubation of sample

1. Grow bacteria in liquid media or on solid plates. Select the type of medium, growth conditions (e.g., temperature, availability of oxygen) and growth time according to the specific requirements of the species of bacteria under investigation. For example, for $S$. aureus Heart Infusion (HI) agar plates can be used, with growth for $16 \mathrm{~h}$ in $37^{\circ} \mathrm{C}$ in aerobic conditions. NOTE: To achieve the best results, the growth/ incubation should yield enough bacteria that would allow the collection of a micro-pellet of sample. The specific number of colony forming units or bacterial cells depend on the type and size of the bacterium.

2. Sample deposition 
1. Using a sterile loop, carefully collect bacteria from the colonies on the agar plate and transfer them to a glass tube. Collect bacteria only from the top of the colonies. If collecting samples from a liquid culture, using a pipette, transfer approximately $1 \mathrm{~mL}$ of the bacterial suspension to a glass tube. The volume can be modified depending on the bacterial load.

NOTE: It is important to attempt to not collect (or minimalize as much as possible the collection of) any medium from underneath the colony. The subsequent steps of sample preparation aim to remove any potential residual of media. Minimization of the potential medium residual from the beginning enables the spectral acquisition of data from purified bacterial cells. Steps 2.2.2 and 2.2.3 apply to samples prepared from agar plates. For samples prepared from liquid media, move to step 2.2.4.

2. Add $1 \mathrm{~mL}$ of ultrapure water to the tube. Vortex until the collected bacterial pellet are no longer visible at the bottom of the tube (typically 1-2 min).

3. Estimate the rough turbidity of the solution using, e.g., McFarland standards by visual comparison $^{30}$ between the prepared solution and McFarland standards. If the turbidity of bacterial suspension appears to be very low, add more bacteria from the plate using a sterile loop and vortex again. Repeat until the rough turbidity of the solution is comparable to McFarland standards 0.5 and 1 . This will generally yield a good amount of bacterial pellet.

4. Centrifuge the bacterial suspension at $3,000 \times g$ for 5 min to obtain a pellet.

NOTE: Centrifugation parameters can be modified to obtain bacterial pellet. Caution should be taken if increasing the g-force, to not induce breakage of bacteria (especially in case of Gram-negative bacteria).

5. Using a pipette, gently remove the supernatant from above the pellet. Add $1 \mathrm{~mL}$ of ultrapure water to the tube and vortex to re-suspend the pellet. Subsequently, centrifuge the sample as was done in step 2.2.4.

6. Repeat the washing procedure (steps 2.2.2 and 2.2.4) at least three times. In case of collection of the initial sample from liquid media, repeat the procedure at least four times (media removal followed by three washes).

7. After the final wash, remove the supernatant, add ultrapure water and vortex for at least $2 \mathrm{~min}$. Subsequently, deposit $5 \mu \mathrm{L}$ of the sample on the substrate (e.g., Raman grade $\mathrm{CaF}_{2}$ ).

8. If the desired thickness of the sample is a multilayer of bacteria, leave the sample to air-dry.

9. If the desired thickness is monolayer or individual bacteria, immediately after depositing the sample (step 2.2.7) add between $20-100 \mu \mathrm{L}$ of ultrapure water and mix gently with a pipette tip. Leave to airdry.

NOTE: The exact volume of water can vary between experiments as it is dependent on many factors (e.g., size of the organism, density of the pellet, etc.) and is, therefore, best determined empirically. Preparation of a series of samples with varying volumes of ultrapure water added enables one to select a sample with the desired thickness/density of bacteria. The thickness/ density of bacteria can be easily visualized via AFM in the subsequent stages. Examples of AFM images 
from monolayer and single cell samples are shown in

Figure 1A-H.

10. Mount the substrate on an AFM metal specimen disk using double-sided adhesive tape.

\section{Instrument preparation}

NOTE: The instrumental procedures described here are for the instrument listed in the Table of Materials. The detail instrumental procedure may differ slightly from the one described here if using a newer model of the AFM-IR instrument.

1. Switch on and initialize the instrument by pressing the Initialize button. Ensure that the laser shutter is in the Open position for the laser test.

2. If a purging system is set up, purge the instrument with $\mathrm{N}_{2}$ by turning on the flow of $\mathrm{N}_{2}$. Adjust the nitrogen purge to achieve a stable humidity level (for example, $20 \%$ ). Ensure that the humidity does not fluctuate during measurements and between background and sample data collection. Allowing approximately $20 \mathrm{~min}$ for the humidity levels to stabilize is recommended.

3. Load the sample into the sample chamber by pressing the Load button. Sample loading is conducted through the software wizard. While operating the software wizard, first focus on the tip, using arrows to move the microscope stage in the Z-direction and click on Next. Secondly, adjust the data collection spot, using arrows guiding the in-plane movement and align the AFM laser and AFM detector using the knobs on the top of the AFM head. Subsequently, focus on the sample surface by moving the microscope stage in Z-direction.

NOTE: Detailed illustrations of each step of sample loading are provided in the software manual ${ }^{31}$. Focusing on the sample should be conducted with care. When approaching the sample surface in the Z-direction, use a slow motor speed.

4. Approach the sample without engaging by clicking on the Approach button.

\section{Data collection}

1. Background

1. Prior to data acquisition, collect the background. For background collection, ensure that the laser shutter is in the Open position. Select the spectral range and resolution (depending on the aim of the analysis) and the number of scans and number of co-averages of background. These are generally recommended to be high (e.g., 1024 scans and 3 co-averages).

NOTE: In general, spectral resolution of $4 \mathrm{~cm}^{-1}$ or 8 $\mathrm{cm}^{-1}$ and spectral ranges of $3,200 \mathrm{~cm}^{-1}-2,800 \mathrm{~cm}^{-1}$ and $1,800 \mathrm{~cm}^{-1}-900 \mathrm{~cm}^{-1}$ are recommended.

2. After acquisition of the background, save the background file. The file is not stored automatically. Change the laser shutter position to Close.

2. Sample - single spectra

1. Press the Engage button to engage to the sample. The system will begin to approach the sample surface, until direct contact is detected.

NOTE: Set point used in this work ranged between $0.15-2 \mathrm{~V}$ and the feedback gains (I Gain and P Gain) would typically be set to 3 and 10 . NIR2 contact probes are commonly used with nanolR2 system (model: PR-EX-nIR2-10, resonance frequency $(\mathrm{kHz})$ : $13+/-4 \mathrm{kHz}$, spring constant (N/m): 0.07-0.4 $\mathrm{Nm}^{-1}$ ). 
2. Collect an AFM image to visualize the surface. In the first instance, scan a larger area (e.g., $50 \times 50 \mu \mathrm{m}$ ) with lower spatial resolution (e.g., $200 \times 200$ points) (Figure 1I).

NOTE: AFM-IR data is always collected in contact mode, however, the AFM data can be collected in contact or tapping mode.

3. From the AFM height/deflection image, select a specific area of interest and re-image it with higher spatial resolution (Figure $\mathbf{1} \mathbf{J}-\mathbf{K}$ ). Ensure that the speed of data collection is appropriate, with slow tip movement (e.g., Scan Rate 0.2-0.4 Hz).

4. Select the measurement spot (e.g., single bacterium) and move the tip to the spot.

5. Align the IR laser. For this purpose, use a wavenumber at which the sample will absorb. For biological materials this can be, e.g., amide I (1655 $\mathrm{cm}^{-1}$ ). Make sure that the Band Pass Filter is off and click on Start IR. The right graph in the nanolR meter (FFT of the deflection displayed as amplitude vs. frequency) should show at least one clear peak and the left graph (deflection vs. time) should have a periodic waveform. If this is not the case, proceed to optimize the IR spots.

NOTE: Even if the Fast Fourier Transform (FFT) and deflection show expected profile, it is recommended to conduct the optimization of IR spots for at least several wavenumbers, where bands are expected.

6. Optimize the hot spots for the IR data collection using the selected wavenumber values. It can be helpful to use a conventional IR spectrum of the bacteria (e.g., ATR spectrum of bacterial pellet) to identify the positions of the bands and use them to optimize the hot spots. Select various wavenumber values (e.g., 8-10) from various spectral regions.

NOTE: If conventional IR spectrum of bacteria of interest could not be collected prior to AFM-IR data collection, bacterial spectra available in the literature can be used as a rough guidance. The outcome of the optimization of an IR spot is an image, that presents a map of the FFT magnitude signal at each $\mathrm{x}$ and $\mathrm{y}$ location. The location with largest signal is selected automatically. Examples of such images are given in software manual ${ }^{31}$.

7. After optimizing the IR spots for selected wavenumber values, define the parameters of spectral data collection: spectral region, spectral resolution, number of scans, and applied power and input these into the appropriate windows in the software. The spectral resolution should match the background resolution and the spectral region should be within the spectral region for which background was collected.

NOTE: A generic initial set of parameters could be: spectral range: $3200 \mathrm{~cm}^{-1}-2800 \mathrm{~cm}^{-1}$ and 1800 $\mathrm{cm}^{-1}-900 \mathrm{~cm}^{-1}$, spectral resolution: $4 \mathrm{~cm}^{-1}$ or 8 $\mathrm{cm}^{-1}$, number of scans: $512-2048$.

8. If needed, adjust the laser power depending on the signal. In general, values between $8 \%-10 \%$ of laser power should be sufficient for good quality of signal. Higher values can be used with caution, as they may result in sample damage.

NOTE: The percent laser power can vary depending on the type of IR laser. The percent values given here are for OPO laser

9. Click on Acquire to collect AFM-IR spectrum. 
10. Re-collect the AFM data from the same area after collection of the AFM-IR spectrum. This is highly recommended as it will reveal any potential drift and/ or destructive influence on the sample.

11. If the AFM-IR spectrum is satisfactory and no destructive influence on the sample is observed, proceed with data collection. If needed, define a series of points for data collection using the Array option and collected AFM height or deflection image. This option allows to collect spectra consecutively from each point with the same spectral parameters as defined for a single spectrum.

12. If the AFM image collected after collection of AFM-IR spectrum reveals destructive influence on the sample (typically a burned spot), reduce the power; select a different spot and repeat steps 4.3.8-4.3.11.

13. If the signal in AFM-IR spectrum is not satisfactory, check the correctness of optimization of IR spots (step 4.3.6). If it is correct, increase the laser power slightly and repeat steps 4.3.7-4.3.11. This can be repeated until satisfactory signal is achieved.

3. Sample - imaging approach

NOTE: It is highly recommended to record a single AFM-IR spectrum of the bacterium prior to collecting an intensity distribution image for a selected wavenumber value.

1. Record an AFM image of the chosen sample area. To do this, first collect AFM image of a larger area with lower spatial resolution (e.g., 50 x $50 \mu \mathrm{m}, 200$ x 200 points), then select a region of interest and collect an AFM image with increased spatial resolution (as illustrated in Figure 1I-K).

2. Select the wavenumber values for AFM-IR imaging.
3. Ensure that the IR spot of the laser is optimized for the selected wavenumber values (step 4.3.6). If the IR spot is not optimized for some wavenumbers (no clear maximum), optimize it for them.

4. Define the parameters of the imaged area: the width and height, number of data points in $X$ and $Y$ direction. NOTE: If the consecutive selection of spots from the previous AFM images is applied (as demonstrated in Figure 1I-K), the width and height fields will be automatically filled up, upon marking of the area.

5. Define the parameters of spectral signal acquisition: the wavelength, number of scans, and laser power. NOTE: The number of scans needs to be kept within reason. 64 or 32 scans will typically allow a sufficient amount of signal.

6. Define the parameters of AFM tip movement by clicking on the Scan Rate. The higher the number of scans in the previous step and the number of data points in $X$ direction, the slower will the tip movements need to be. Lack of adjustment between these parameters will result in too fast movement of the tip, preventing the actual acquisition of defined number of scans from each point.

NOTE: For example, for an appropriate collection of IR signal with 64 co-additions and 200 points, set the Scan Rate as $0.07 \mathrm{kHz}$.

7. Make sure that the Enable IR imaging box is ticked.

8. Begin imaging. AFM-IR of the intensity of signal at the selected wavenumber will be collected simultaneously with the AFM data from that area.

NOTE: When the OPO laser is used, it is possible to additionally collect simultaneously contact resonance peak frequency image. This can be used to obtain 
information about the relative stiffness of the sample at different locations.

9. Use the Capture Sequence window to set a consecutive collection of AFM-IR data from the same area with the same parameters, but for different wavenumber values. To do that, open the Capture Sequence window, type in each wavenumber, and define the applied laser power (for each wavenumber).

10. Export the collected data (AFM and AFM-IR, single spectra and imaging) into various formats and analyze it using methods adequate for specific research aims.

\section{Representative Results}

The described protocol enables to obtain a range of types of cell distributions of bacteria on the substrate, depending on the initial concentration of sample and the amount of water added. Figure 1 illustrates the examples of AFM images (height and deflection) recorded from monolayers and singlecell samples prepared using the described protocol from Gram-positive (S. aureus) and Gram-negative (Escherichia coli) bacteria.

The protocol described here can be utilized for AFM-IR imaging of intra- and extra-cellular structures of single bacteria. An example of this application is shown in Figure 2, which demonstrates the results of monitoring the spatially localized chemical changes occurring during division of an S. aureus cell. Although air-drying is commonly considered as a fixation approach for bacterial preparation, bacteria, by nature, show very high resistance to external factors such as temperature and were reported to survive dehydration ${ }^{32}$. The results presented here were acquired from an air- dried sample. The formation of a septum, occurring prior to cell division was observed and monitored via AFM imaging (Figure 2A-D) by collection of 12 images of the same area consecutively (collection of a single image $\approx 20 \mathrm{~min}$ ). Figure 2A-D shows 4 selected AFM images, with time between collection of each image of approximately $40 \mathrm{~min}$. The formed structure (septum) is $45 \mathrm{~nm}$ high. The formed septum is clearly visible in AFM height and deflection images (Figure 2E-F). The AFM-IR spectra recorded from the cell and septum area (Figure 2G, points of origin marked in Figure 2F) were normalized to the amide I band prior to comparison, to minimalize the influence of varying sample thickness between points of data collection. The AFM-IR spectrum of the septum is characterized by higher relative intensity of bands at 1240 and $1090 \mathrm{~cm}^{-1}$ compared to AFM-IR spectrum collected from cell area. These are attributed to carbohydrate and phosphodiester groups of cell wall components (including e.g., peptidoglycan and teichoic acid) ${ }^{22}$.

The described protocol can also be used for comparison of a single spectra between a number of different samples. An example of this application together with the results is shown in Figure 3 and Figure 4. The aim of the study is to determine the chemical changes occurring as a result of in vivo development of vancomycin intermittent resistance in S. aureus (VISA). For this purpose, clinical pairs of samples were collected from patients, with the parent strain isolated upon admission to the hospital and prior to antibiotic therapy (vancomycin susceptible S. aureus, VSSA) and the daughter strain isolated from the same patient after admission of antibiotics and clinical failure. Samples were further grown on agar medium and prepared according to the protocol (Figure 3A-B). The AFM-IR spectra were collected from multiple single bacteria (and multiple samples) for VSSA and 
VISA and subsequently analyzed using several chemometric approaches (Figure $3 \mathbf{C}$ ).

No morphological differences were observed between VSSA and VISA cells (Figure 4A-C). However, the AFM-IR spectra (Figure 4D,F) and their second derivatives (Figure 4E,G) demonstrated a clear difference in the chemical composition between resistant and susceptible strains. The relative intensity of the bands associated with carbohydrate and phosphodiester groups from cell wall components (in particular, the band at $1088 \mathrm{~cm}^{-1}$ ) clearly increased in the resistant strain, compared to the susceptible counterpart. Of note is the fact that all recoded spectra (VISA: 81, VSSA: 88) show a small standard deviation. This demonstrates good reproducibility of data recorded from various samples prepared from the same strain, as no discrimination was possible between spectra recorded from different samples of the same strain. The observed differences indicated an increased thickness of the cell wall in resistant strains, compared to the susceptible counterpart, which remains in agreement with other literature reports ${ }^{33,34}$. 

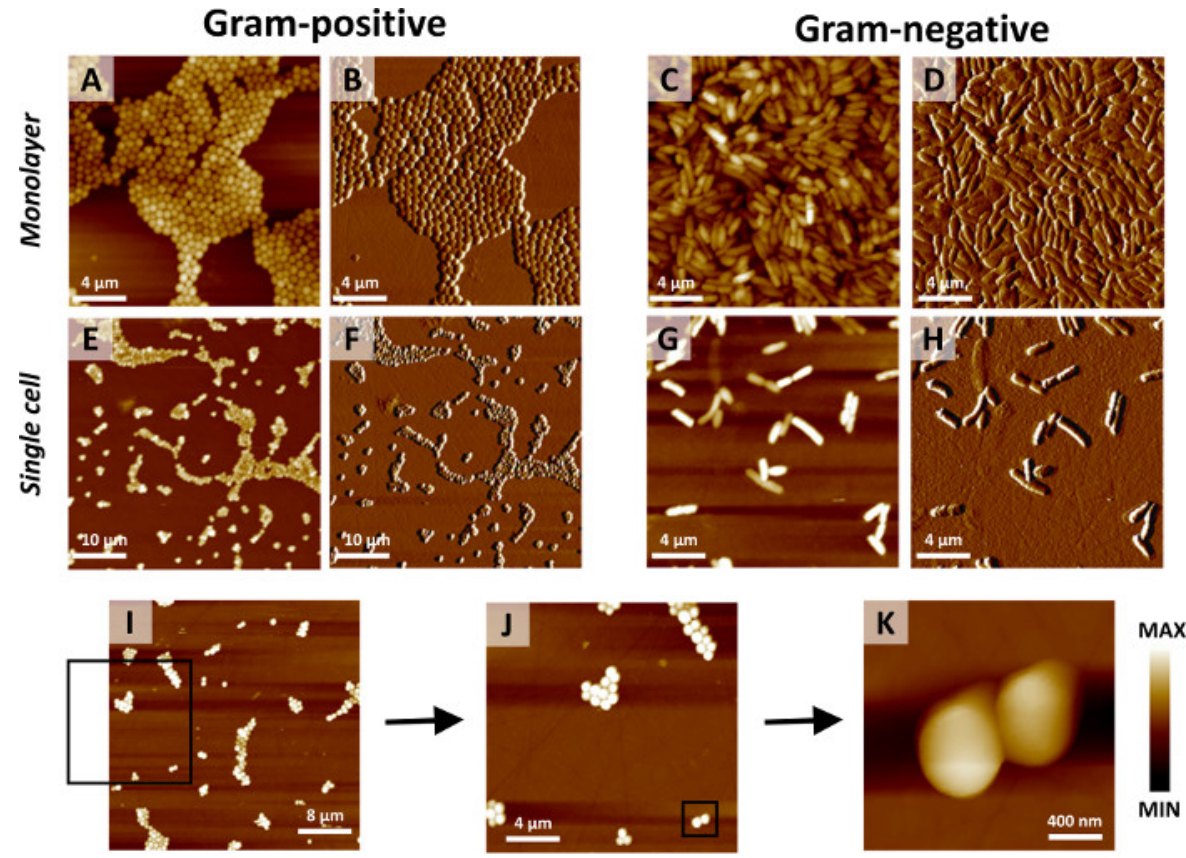

Figure 1: Representative AFM images of various bacterial samples for AFM-IR measurements. Depending on the dilution on substrate, the protocol allows one to obtain multilayers and monolayers of bacteria as well as single-cell samples. Representative AFM images of: (A-D) monolayer and (E-H) single-cell sample for (A,B,E,F) Gram-positive (S. aureus) and $(\mathbf{C}, \mathbf{D}, \mathbf{G}, \mathbf{H})$ Gram negative (E. coli) bacteria. (A,C,E,G) demonstrate height images and (B,D,F,H) show corresponding deflection images. Size of imaged areas: $(\mathbf{A}-\mathbf{D}, \mathbf{G}, \mathbf{H}) 20 \times 20 \mu \mathrm{m},(\mathbf{E}, \mathbf{F}) 50 \times 50 \mu \mathrm{m}$. (I-K) consecutive selection of an area for AFM-IR mapping. This is achieved using AFM imaging with increasing spatial resolution in the example of a single $S$. aureus cell. Each image is collected by sampling $200 \times 200$ points, with increasing spatial resolution due to the reduction in the size of the imaged area. Size of imaged areas: (I) $40 \times 40 \mu \mathrm{m},(\mathrm{J}) 20 \times 20 \mu \mathrm{m}$ and (K) $2.24 \times 2.24 \mu \mathrm{m}$. The black square in (I) marks the area imaged in $(\mathbf{J})$. The black square in $(\mathbf{J})$ marks the area imaged in $(\mathbf{K})$. Please click here to view a larger version of this figure. 

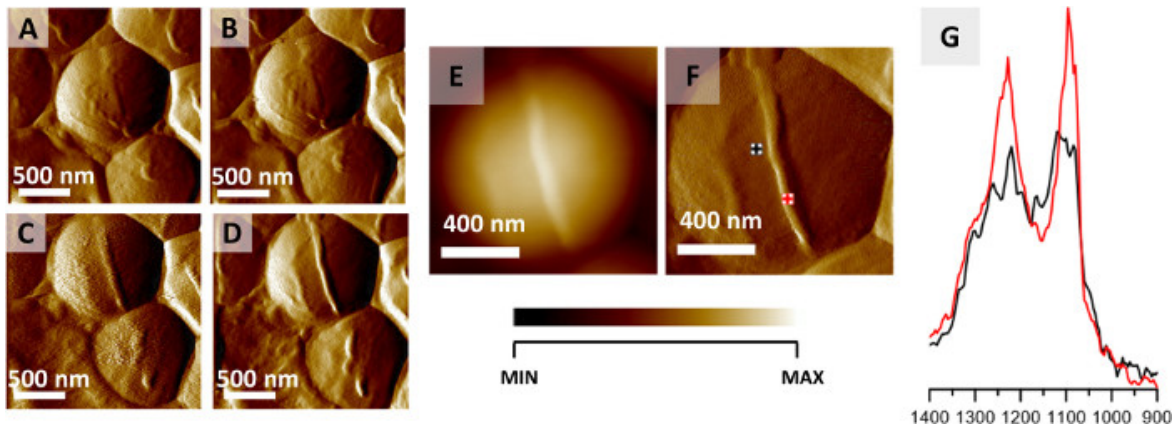

Figure 2: Monitoring $S$. aureus cell division via AFM-IR. (A-D) AFM images of $S$. aureus cell showing the formation of septum preceding cell division. Size of imaged area: $2 \times 2 \mu \mathrm{m}$. The images were selected from a larger series (12 images recorded every $20 \mathrm{~min}$ ) and represent data recorded every $40 \mathrm{~min}$. (E-F) AFM height and deflection image recorded at the end of cell septum formation with marked points of collection of AFM-IR spectra. Size of the imaged area $1.17 \times 1.15 \mu \mathrm{m}$. The height of the newly formed structure is $45 \mathrm{~nm}$. (G) AFM-IR spectra recorded from cell area (black) and septum area (red) (marked in $(\mathbf{F})$ ), in the range $1400-900 \mathrm{~cm}^{-1}$. Both spectra were normalized to the amide I band and demonstrate an increase in the relative intensity of cell wall components from the septum. This figure has been modified from K. Kochan et al. $^{22}$. Please click here to view a larger version of this figure. 


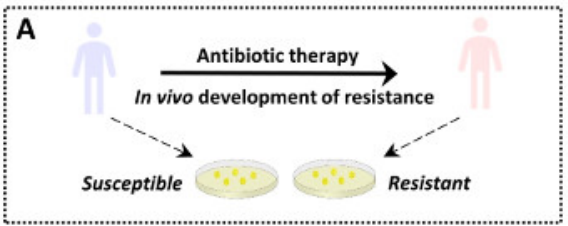

B
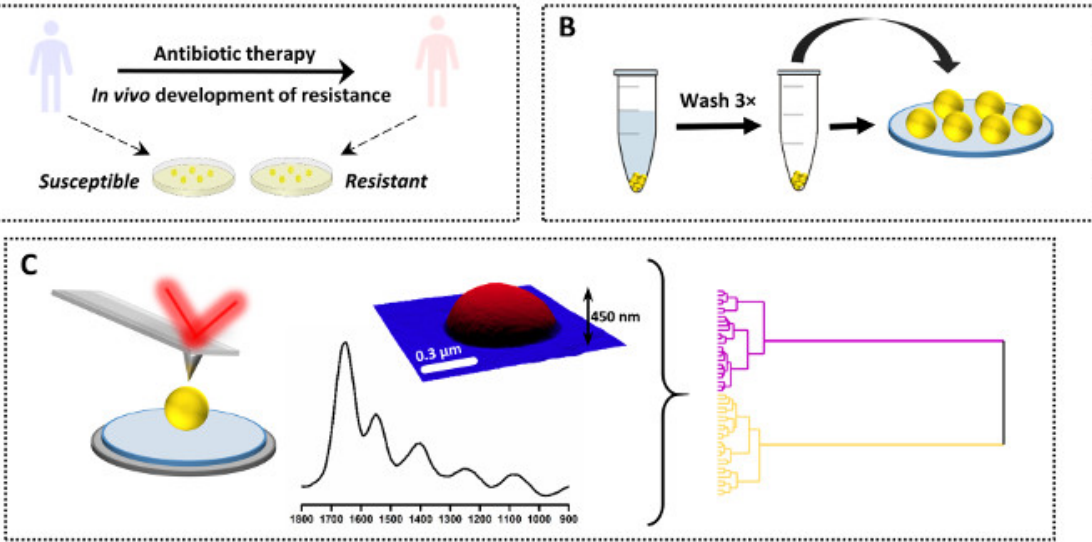

Figure 3: An overview of the experimental design for AFM-IR study of antimicrobial resistance. (A) Sample origin and initial preparation: susceptible parent strain was collected from a patient prior to antibiotic therapy and the daughter resistant strain was sourced from the same patient after antibiotic therapy and clinical failure (in vivo resistance development). Bacteria were isolated and cultured on Heart Infusion $(\mathrm{HI})$ agar for $16 \mathrm{~h}$ in $37^{\circ} \mathrm{C}$. (B) Subsequent sample preparation for AFM-IR, including collection of the sample followed by washing of bacterial pallet ( $3 \times$ ) and sample deposition. (C) AFMIR data collection and analysis: AFM height and AFM-IR spectrum (1800-900 $\left.\mathrm{cm}^{-1}\right)$. Size of the AFM imaged area: 1.7 x $1.4 \mu \mathrm{m}$. The AFM-IR spectrum was collected from the middle of the cell. The data was subsequently analyzed using chemometric approaches, including hierarchical cluster analysis. This figure has been modified from K. Kochan et al. ${ }^{19}$. Please click here to view a larger version of this figure. 

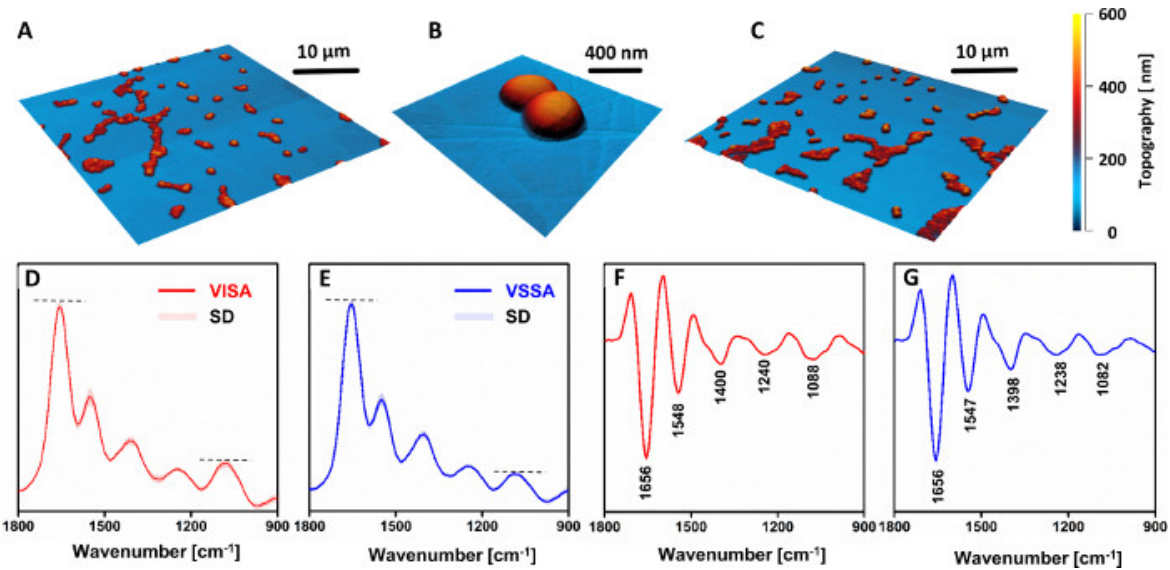

Figure 4: AFM and AFM-IR results of study of chemical changes in vancomycin intermediate S. aureus (VISA) compared to vancomycin susceptible $S$. aureus (VSSA) in clinical pairs. AFM images of (A-B) VISA and (C) VSSA single-cell samples. Size of the imaged areas: (A,C) $40 \times 40 \mu \mathrm{m}$, (B) $2.56 \times 2.45 \mu \mathrm{m}$. (D-E) Average AFM-IR spectra and their $(\mathbf{F}-\mathbf{G})$ second derivatives for: $(\mathbf{D}, \mathbf{F})$ VISA and $(\mathbf{E}, \mathbf{G})$ VSSA cells, in the spectral range $1800-900 \mathrm{~cm}^{-1}$. The spectra presented are an average of 81 (VISA) and 88 (VSSA) individual spectra and are presented together with standard deviation (SD). The averaging was conducted after normalization of all individual spectra together. The major bands are marked in (FG). This figure has been modified from K. Kochan et al. ${ }^{19}$. Please click here to view a larger version of this figure.

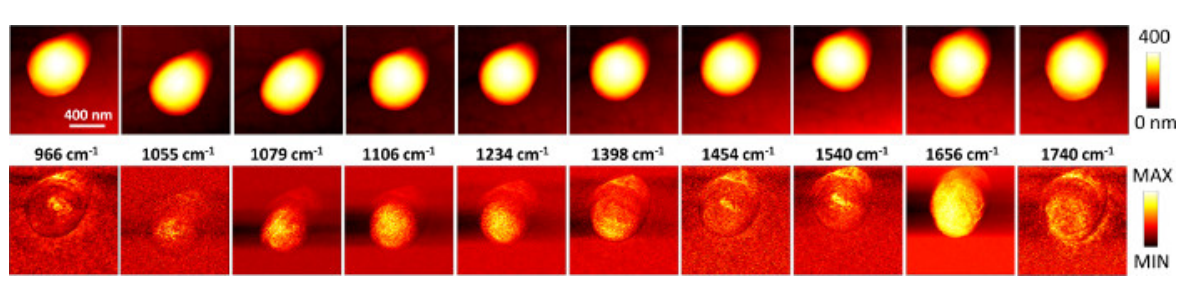

Figure 5: Image drift over consecutive registration of AFM-IR maps at selected wavenumber values for the $S$. aureus cell. (Top row): AFM images were recorded simultaneously with the corresponding (bottom row) AFM-IR maps based on the intensity of the IR signal at selected wavenumber values. The wavenumber values $(966,1055,1079,1106,1234,1398$, $1454,1540,1656,1740 \mathrm{~cm}^{-1}$ ) are annotated above the bottom row. Each set (AFM image and AFM-IR map) was recorded directly after the previous image (approximately $40 \mathrm{~min}$ per set). The size of the imaged/mapped area: $1.54 \times 1.57 \mu \mathrm{m}$. A clear drift is visible between the images. Please click here to view a larger version of this figure. 


\section{Discussion}

The usefulness of IR spectroscopy for characterization of a broad range of biological samples in the context of their chemical composition is well established. Over the past decade, IR spectroscopy has emerged as a promising tool for bacterial studies ${ }^{12}, 13,14,15,16,17$. It continues to attract substantial interest in the field of microbiology, as one of the few techniques enabling a phenotypic characterization through the chemical composition. In this context, the major drawback of conventional FTIR microscopy lies in limited spatial resolution, preventing single cell and subcellular studies of bacteria. In fact, the small size of bacteria represents an impediment not only for IR, but for the vast majority of techniques. Thus, the available research tools for single cell and subcellular studies of bacteria are significantly limited. The combination of AFM with IR enables the spatial resolution limitation of IR spectroscopy to be overcome, providing a novel tool for bacterial research, capable of nanoscale probing of the chemical composition.

The technique is not limited to single cell studies and allows one to probe a variety of samples, ranging in thickness. Undoubtedly, clean, and careful sample preparation is critical to achieving high quality images. The protocol herein provides a method to prepare multilayer, monolayer, and/or single cell samples of various bacteria (Figure 1). The prepared sample depends on several factors, including the initial bacterial load, post-washing dilution as well as further dilution on the substrate. The amount of sample obtained after diluting the washed pellets and prior to deposition on the substrate typically allows the preparation of numerous samples. Therefore, to obtain the desired distribution of the sample on the substrate, it is often beneficial to prepare a series of samples, ranging in their dilution. For studies aiming at collection of AFM-IR spectra rather than subcellular imaging, modifying the amount of sample (e.g., from monolayer to multilayer) may be beneficial to increase the intensity of the signal.

Another critical aspect in sample preparation is appropriate removal of medium residuals. Depending on the selected sample culturing methods, the sample is collected either from liquid medium or from an agar plate. In both cases, the medium residual is likely to be present in the sample, although to a much lesser extent upon collection from agar plates. As bacterial growth media contain an abundance of various biological components, it is critical to ensure appropriate removal of medium. We recommend three washes with ultrapure water for agar plate samples and at least four washes for samples collected from medium. The number of washes can be increased, if needed; however, for comparison between various samples, it is important to keep it consistent between samples. The demonstrated protocol utilizes water, rather than solvents such as phosphate buffer solution (PBS) or saline. Both PBS and saline lead to formation of crystals upon air-drying, which can damage the bacteria. In addition, both are a source of intense IR bands, with PBS, in particular, containing multiple bands in the fingerprint region. The lack of ability for the use of saline or PBS, currently represents an important limitation for the technique. Typically, the use of water for washing does not cause any destructive influence on the bacteria; however, care should be taken, and if possible, the time of water exposure should be limited. If the sample preparation protocol needs to be paused at the stage of washing, it is recommended to leave the sample in pelletized form after removing the water. This is of particular importance for Gram-negative bacteria, containing a thinner cell wall as they are more prone to rupture. 
To ensure proper and high-quality AFM-IR data, several aspects in data collection protocol are of critical importance. Firstly, the correct collection of background is essential for data acquisition. In particular, maintenance of stable humidity levels throughout background collection as well as between background and sample collection is necessary. To ensure this, we recommend purging the instrument with nitrogen and maintaining the humidity levels not higher than $25 \%$. Lack of purging can impose a significant limitation, particularly in places with high humidity. Secondly, the importance of proper optimization of IR spots should be highlighted. For best results, a priori knowledge about the position of band maxima can be beneficial. For example, a conventional IR spectrum of bacterial pellet can be used to determine positions of bands expected from a sample. If that is not possible to acquire, as an alternative approach, the user can utilize IR spectra available in literature or begin the optimization using a band position that is reasonable to expect in the bacterium (e.g., amide I and amide II). Thirdly, for data collection, it is important to highlight the significance of careful power selection (allowing to achieve a good $\mathrm{S} / \mathrm{N}$ ratio), as it can have a destructive effect. The advised power depends on the thickness of the sample, with rough guidance available in the instrument manual ${ }^{31}$. We recommend to empirically test the state of the sample post-measurement by collection of an AFM image, as it will reveal any destructive influence. Furthermore, the collection of AFM images from the same area before and after collection of AFM-IR spectra serves as a good confirmation that no drift has occurred and the spectra indeed originate from the selected point in the cell. The possibility of drift is particularly important when applying the imaging modality, through consecutive imaging of IR intensity at selected wavenumber values. An example of this is illustrated in Figure 5. The imaged area was defined at the beginning of the experiment and is meant to be consistent for all wavenumber values. However, a clear drift is visible between each AFM height (and the corresponding IR wavenumber intensity) image, with acquisition time of each map of approximately $40 \mathrm{~min}$. Due to this, for users collecting imaging data, we recommend to always select an area slightly larger than the sample of interest, to ensure that even upon existence of drift, the sample of interest will remain within the imaged area.

The potential limitations of the protocol include the lack of ability to collect data in a hydrated state in physiological solutions (e.g., saline or PBS) described above. Moreover, especially in high humidity areas, there is often a need for nitrogen purge. Furthermore, the protocol enables to probe organisms down to $100 \mathrm{~nm}$ in size, excluding the possibility of its use for smaller structures. Although this can be overcome using a different laser (e.g., quantum cascade laser allowing to achieve the spatial resolution of $20 \mathrm{~nm}$ ), it is also associated with limited spectral range as well as difficulties in obtaining a good signal to noise ratio. Finally, probing of soft surfaces may present a challenge with the tip not detecting the surface properly and proceeding beyond the point of contact, until breakage. Although this is typically not an issue with bacterial samples, it may occur upon measurements of softer samples. In such cases, it is recommended to attempt to engage on clean surface of the substrate in proximity to the sample.

The described protocol can be utilized for numerous types of bacterial research, including comparative studies between various samples as well as subcellular examination. The data can be analyzed using chemometric approaches for single spectra and imaging modalities ${ }^{35}$, depending on the aim of the research. Furthermore, the protocol can also be modified for application to other biological material (such as fungi, yeast, cells, etc.), through addition of fixation. 


\section{Disclosures}

We would like to acknowledge Bruker for the payment of publication fee. $\mathrm{KK}, \mathrm{BRW}, \mathrm{AP}$, and $\mathrm{PH}$ are inventors on an international patent (PCTIB2020/052339), which describes some of the fundamental aspects of the approach.

\section{Acknowledgments}

We would like to acknowledge Bruker for their support. This work was supported by Monash University Advancing Women's Success Grant (K. Kochan). A.Y.P acknowledges support from an Australian National Health and Medical Research Council Practitioner Fellowship (APP1117940). This work was funded by an Australian Research Council Discovery Project DP180103484. We would like to thank Mr. Finlay Shanks for his instrumental support and Ms. Xenia Kostoulias for her technical assistance with the samples.

\section{References}

1. Sears, C. L. A dynamic partnership: celebrating our gut flora. Anaerobe. 11 (5), 247-251 (2005).

2. Rudd, K. E. et al. Global, regional, and national sepsis incidence and mortality, 1990-2017: analysis for the Global Burden of Disease Study. The Lancet (London, England). 395 (10219), 200-211 (2020).

3. Seymour, C. W. et al. Time to treatment and mortality during mandated emergency care for sepsis. The New England Journal of Medicine. 376 (23), 2235-2244 (2017).

4. Weiss, S. L. et al. Delayed antimicrobial therapy increases mortality and organ dysfunction duration in pediatric sepsis. Critical Care Medicine. 42 (11), 2409-2417 (2014).

5. Peker, N., Couto, N., Sinha, B., Rossen, J. W. Diagnosis of bloodstream infections from positive blood cultures and directly from blood samples: recent developments in molecular approaches. Clinical Microbiology and Infection: The Official Publication of the European Society of Clinical Microbiology and Infectious Diseases. 24 (9), 944-955 (2018).

6. Aminov, R. I. The role of antibiotics and antibiotic resistance in nature. Environmental Microbiology. 11 (12), 2970-2988 (2009).

7. Levy, S. B., Marshall, B. Antibacterial resistance worldwide: causes, challenges and responses. Nature Medicine. 10 (12 Suppl), S122-129 (2004).

8. Roca, I. et al. The global threat of antimicrobial resistance: science for intervention. New Microbes and New Infections. 6, 22-29 (2015).

9. O'Neill, J. The review on antimicrobial resistance. Tackling drug-resistant infections globally: final report and recommendations. Wellcome Trust. (2016).

10. Perry, J. A., Westman, E. L., Wright, G. D. The antibiotic resistome: what's new? Current Opinion in Microbiology. 21, 45-50 (2014).

11. Piddock, L. J. Assess drug-resistance phenotypes, not just genotypes. Nature Microbiology. 1 (8), 16120 (2016).

12. Naumann, D., Helm, D., Labischinski, H. Microbiological characterizations by FT-IR spectroscopy. Nature. 351 (6321), 81-82 (1991).

13. Zarnowiec, P., Lechowicz, L., Czerwonka, G., Kaca, W. Fourier Transform Infrared Spectroscopy (FTIR) as a tool for the identification and differentiation of pathogenic bacteria. Current Medicinal Chemistry. 22 (14), 1710-1718 (2015).

14. Quintelas, C., Ferreira, E. C., Lopes, J. A., Sousa, C. An overview of the evolution of infrared spectroscopy 
applied to bacterial typing. Biotechnology Journal. 13 (1), 1700449 (2018).

15. San-Blas, E., Cubillán, N., Guerra, M., Portillo, E., Esteves, I. Characterization of xenorhabdus and photorhabdus bacteria by Fourier transform mid-infrared spectroscopy with attenuated total reflection (FT-IR/ATR). Spectrochimica Acta. Part A, Molecular and Biomolecular Spectroscopy. 93, 58-62 (2012).

16. Sousa, C. et al. Discrimination of the acinetobacter calcoaceticus-acinetobacter baumannii complex species by Fourier transform infrared spectroscopy. European Journal of Clinical Microbiology \& Infectious Diseases. 33 (8), 1345-1353 (2014).

17. Rodriguez-Saona, L. E., Khambaty, F. M., Fry, F. S., Calvey, E. M. Rapid detection and identification of bacterial strains by Fourier transform nearinfrared spectroscopy. Journal of Agricultural and Food Chemistry. 49 (2), 574-579 (2001).

18. Dawson, S. E. et al. Implementation of Fourier transform infrared spectroscopy for the rapid typing of uropathogenic Escherichia coli. European Journal of Clinical Microbiology \& Infectious Diseases. 33 (6), 983-988 (2014).

19. Kochan, K. et al. Detection of Antimicrobial ResistanceRelated Changes in Biochemical Composition of Staphylococcus aureus by Means of Atomic Force Microscopy-Infrared Spectroscopy. Analytical Chemistry. 91 (24), 15397-15403 (2019).

20. Dazzi, A., Prater, C. B. AFM-IR: Technology and Applications in Nanoscale Infrared Spectroscopy and Chemical Imaging. Chemical Reviews. 117 (7), 5146-5173 (2017).
21. Dazzi, A. et al. AFM-IR: combining atomic force microscopy and infrared spectroscopy for nanoscale chemical characterization. Applied Spectroscopy. 66 (12), 1365-1384 (2012).

22. Kochan, K. et al. In vivo atomic force microscopy-infrared spectroscopy of bacteria. Journal of the Royal Society, Interface. 15 (140) (2018).

23. Katzenmeyer, A. M. et al. Mid-infrared spectroscopy beyond the diffraction limit via direct measurement of the photothermal effect. Nanoscale. 7 (42), 17637-17641 (2015)

24. Bruker Life Science Applications. https:// www.bruker.com/products/surface-and-dimensionalanalysis/nanoscale-infrared-spectrometers/nanoscale-irspectroscopy-applications/life-sciences.html (2020).

25. Mayet, C., Dazzi, A., Prazeres, R., Ortega, J. M., Jaillard, D. In situ identification and imaging of bacterial polymer nanogranules by infrared nanospectroscopy. Analyst. 135 (10), 2540-2545 (2010).

26. Baldassarre, L. et al. Mapping the amide I absorption in single bacteria and mammalian cells with resonant infrared nanospectroscopy. Nanotechnology. 27 (7), 075101 (2016).

27. Vitry, P. et al. Combining infrared and mode synthesizing atomic force microscopy: Application to the study of lipid vesicles inside Streptomyces bacteria. Nano Research. 9 (6), 1674-1681 (2016).

28. Dazzi, A. et al. Chemical mapping of the distribution of viruses into infected bacteria with a photothermal method. Ultramicroscopy. 108 (7), 635-641 (2008).

29. Steenbergen, J. N., Alder, J., Thorne, G. M., Tally, F. P. Daptomycin: a lipopeptide antibiotic for the treatment 
of serious Gram-positive infections. The Journal of Antimicrobial Chemotherapy. 55 (3), 283-288 (2005).

30. Garcia, L. S. MacFarlan Standards, in Clinical Microbiology Procedures Handbook, 3rd Edition. American Society of Microbiology. 5.14.1 (2010).

31. Anasys Instruments. NanolR-2 System Manual. https:// www.anasysinstruments.com/downloadpr/ nanolR2_s_System_Manual.pdf. (2020).

32. Whelan, D. R. et al. Detection of an en masse and reversible B- to A-DNA conformational transition in prokaryotes in response to desiccation. Journal of the Royal Society, Interface. 11 (97), 20140454 (2014).

33. McGuinness, W. A., Malachowa, N., DeLeo, F. R. Vancomycin Resistance in Staphylococcus aureus. The Yale Journal of Biology and Medicine. 90 (2), 269-281 (2017).

34. Howden, B. P., Peleg, A. Y., Stinear, T. P. The evolution of vancomycin intermediate Staphylococcus aureus (VISA) and heterogenous-VISA. Infection, Genetics and Evolution: Journal of Molecular Epidemiology and Evolutionary Genetics in Infectious Diseases. 21, 575-582 (2014).

35. Perez-Guaita, D. et al. Multispectral Atomic Force Microscopy-Infrared Nano-Imaging of Malaria Infected Red Blood Cells. Analytical Chemistry. 90 (5), 3140-3148 (2018). 